\title{
MedienPädagogik
}

Zeitschrift für Theorie und Praxis der Medienbildung

\section{Digitale Medien in der Grundschullehrerbildung}

\section{Erfahrungen aus dem Projekt dileg-SL}

Horst Niesyto und Thorsten Junge

\section{Zusammenfassung}

Im Rahmen des Verbundprojekts der Deutsche Telekom Stiftung «Digitales Lernen Grundschule» fand an der Pädagogischen Hochschule Ludwigsburg von 2016-2019 das Entwicklungsprojekt «Digitales Lernen Grundschule - Stuttgart/ Ludwigsburg» statt. In acht Teilprojekten kooperierte die Abteilung Medienpädagogik mit den Fächern Biologie, Deutsch, Englisch, Informatik, Mathematik, Musik und Sport. Über 200 Studierende entwickelten in Hochschulseminaren Konzepte für Unterrichtseinheiten und erprobten diese an der Rosensteinschule in Stuttgart. Das Projekt wurde im Rahmen einer internen Evaluation ausgewertet. Der Beitrag stellt nach einer kompakten Darstellung der Ziele, der theoretischkonzeptionellen, praktischen und evaluativen Dimensionen des Projekts wichtige Beobachtungen und Erfahrungen in teilprojektübergreifender Perspektive vor. Folgende Aspekte werden schwerpunktmässig thematisiert: vorhandene und fehlende Medienkompetenzen der Studierenden; Potenziale visueller und audiovisueller Digitalmedien; reflexive Prozesse bei den Projektaktivitäten. Ein Fazit fasst die wichtigsten Punkte zusammen und skizziert wesentliche Dimensionen einer Grundbildung Medien für die Primarstufenbildung. Der Ausblick betont die Notwendigkeit, eine kritisch-reflexive Medienbildung noch deutlicher von einer «Digitalisierungsoffensive» abzugrenzen, die eine Anpassung an bestehende gesellschaftlich-mediale Machtstrukturen intendiert. 


\title{
Digital media in primary school teacher education. Experiences from the project dileg-SL
}

\begin{abstract}
As part of the Deutsche Telekom Foundation's joint project "Digital Learning Primary School", the development project "Digital Learning Primary School Stuttgart/Ludwigsburg" took place at the Ludwigsburg University of Education from 2016-2019. The Media Education department cooperated in eight subprojects with the academic disciplines Biology, German, English, Computer Science, Mathematics, Music and Sports. More than 200 students developed concepts for teaching units in university seminars and tested them at the Rosenstein primary school in Stuttgart. The project was evaluated as part of an internal evaluation. After a compact presentation of the goals, the theoreticalconceptual, practical and evaluative dimensions of the project, the article presents important observations and experiences from a cross-project perspective. The following aspects will be addressed: existing and missing media competencies of students; potentials of visual and audiovisual digital media; reflective processes during project activities. A conclusion summarises the most important points and outlines essential dimensions of basic media education for primary education. The outlook emphasises the necessity of distinguishing critical-reflexive media education even more clearly from a "digitisation offensive" that aims to adapt to existing social-media power structures.
\end{abstract}

\section{Ausgangsüberlegungen}

Die Grundschule als zentraler Ort einer Grundbildung für alle Kinder steht vor der Aufgabe, Medienbildung in das Bildungsangebot breitenwirksam und verbindlich $\mathrm{zu}$ integrieren. Leider ist die Situation im Grundschulbereich nach wie vor nicht durch breitenwirksame Anstrengungen bei der Verankerung von Medienbildung gekennzeichnet (Deutsche Telekom Stiftung 2014, 22). Da Medienkompetenz weder durch familiale Erziehung

1 Der vorliegende Beitrag ist eine komprimierte Fassung von Fachartikeln, die zur Gesamtauswertung des Ludwigsburger Projekts erschienen (Niesyto 2019a; Niesyto 2019b; Junge 2019). Die Teile 1, 2, 3.2, 3.3 und 5 sind von Horst Niesyto, die Teile 3.1 und 4 von Thorsten Junge. 
noch durch die individuelle Nutzung in der Freizeit allein erworben werden kann, ist jedoch eine grundlegende Medienbildung im Rahmen schulischer Bildung erforderlich, die sowohl auf Persönlichkeitsbildung als auch auf gesellschaftliche Teilhabe abzielt (KBoM 2011; KMK 2012; Breiter et al. 2013). Eine wichtige Voraussetzung hierfür sind nicht nur funktionierende und zuverlässige technische Infrastrukturen an Schulen. Entscheidend sind vielmehr pädagogisch gut ausgebildete Lehrpersonen, die über hinreichende medienpädagogische und mediendidaktische Grundlagen verfügen (Peschel und Irion 2016; Irion und Kammerl 2018; Grundschulverband 2018).

Es ist eine grundsätzliche Position der Medienpädagogik, dass weder eine einseitig technologieorientierte Perspektive noch eine bewahrpädagogische Abwehrhaltung geeignet sind, um Medienbildung in Grundschulen zu befördern. Medienbildung in der Grundschule hat die Aufgabe, die Mediennutzung von Kindern aktiv zu begleiten und pädagogisch-didaktische Impulse für Bildungs- und Lernprozesse mit und über (digitale) Medien zu entwickeln. Im Spannungsfeld von gesellschaftlich-medialen Themen und Angebotsstrukturen, subjektiven Medienpraxen von Kindern und schulischen Bildungskontexten hat eine handlungsorientierte Medienbildung die Chance, medienbezogene Bildungs- und Lernprozesse zu fördern (Kammerl und Irion 2018; Tulodziecki et al. 2019). Die Nutzung (digitaler) Medien führt dabei nicht automatisch zu einem besseren Unterricht, sondern ist stets in Zusammenhang mit jeweils spezifischen (Kontext-) Bedingungen und pädagogisch-didaktischen Szenarien zu betrachten (Herzig 2014).

In diesem Zusammenhang ist darauf hinzuweisen, dass die Schülerschaft an Grundschulen oftmals sehr heterogen zusammengesetzt ist. Dies betrifft insbesondere Faktoren wie die familiale Sozialisation, Begabungen, kognitive Fähigkeiten und den sprachlich-kulturellen Hintergrund. Aus der Mediensozialisationsforschung sind soziokulturelle Unterschiede und soziale Ungleichheiten im Medienhandeln seit langem bekannt (u.a. Kutscher und Otto 2010; Niesyto et al. 2009; Verständig, Klein, und Iske 2016). Befunde aus Praxisforschungsprojekten konnten insbesondere Chancen einer handlungsorientierten Medienarbeit gerade in bildungsbenachteiligten Milieus belegen (Niesyto et al. 2007). Dies betrifft vor allem 
die Integration von Bildern und Bewegtbildern bei der Artikulation von eigenen Themen, Bedürfnissen und Interessen. In didaktischer Perspektive bedeutet dies, Grundsätze wie Lebenswelt- und Handlungsorientierung, Gestaltungsfreiheit und Anschaulichkeit (bei Themenwahl, Ausdrucksform und Arbeitsweise), Balance von Prozess- und Produktorientierung, Zeit für soziales Lernen und Reflexion sowie eine Balance von individuellen und kooperativen Arbeitsformen zu beachten (Niesyto 2010, 399 f.). Gleichzeitig sollten im Sinne einer inklusiven Medienbildung Barrierefreiheit, Ressourcenorientierung und eine Breite an medialen Rezeptions- und Ausdrucksformen gewährleistet sein (Schluchter 2015; 2019).

Um Medienbildung an Grundschulen besser zu verankern, ist es eine wichtige Aufgabe, dass Medienpädagogik, Grundschulpädagogik und die einzelnen Fächer in der 1. Phase der Lehrerbildung in diesem Bereich intensiv kooperieren. Diese Kooperation bezieht sich sowohl auf eine fächerintegrative Medienbildung als auch auf fächerübergreifende Themen- und Kompetenzfelder. Hierzu gehört auch die Erprobung und Integration von pädagogischen Szenarien im Schnittfeld von schulischer und ausserschulischer Medienbildung. Notwendig sind Studienangebote, die für Studierende sowohl theoretische-konzeptionelle Orientierungs- und Reflexionsräume eröffnen als auch praxisbezogene Erprobungsmöglichkeiten in schulischen Kontexten bieten.

Die Hochschulen stehen vor der Herausforderung, allen Lehramtsstudierenden eine Grundbildung Medien zu ermöglichen. Hierfür gibt es seit geraumer Zeit verschiedene Ansätze und erprobte Modelle (Imort und Niesyto 2014), die in den letzten Jahren durch weitere Überlegungen und Rahmenkonzepte ergänzt wurden (siehe u.a. Sektion Medienpädagogik 2017). Das Projektprogramm der Deutsche Telekom Stiftung «Digitales Lernen Grundschule» bot an der PH Ludwigsburg die Gelegenheit, bereits vorhandene Ansätze und Aktivitäten zur Verankerung der Medienbildung und einer Grundbildung Medien (Niesyto 2014; Trüby 2017) in der Primarstufenbildung durch Tandemseminare zwischen der Medienpädagogik und den beteiligten Fächern inhaltlich zu vertiefen und weiterzuentwickeln. 


\section{Ziele und Dimensionen des Projekts dileg-SL}

Im Projekt ging es vor allem um die Frage, wie Studierende im Spannungsfeld von fachbezogenen, medien- und grundschulpädagogischen Überlegungen Kompetenzen für einen kreativen und zugleich reflexiven Einsatz digitaler Medien an Grundschulen erwerben können. Die Projektkonzeption betonte, dass eine pädagogisch-didaktische Grundorientierung intendiert ist, die Themen in der Grundschule nicht ausschliesslich als Perspektive eines Faches, sondern auch interdisziplinär und vor allem aus der Perspektive der Weltaneignung von Kindern begreift (Deckert-Peaceman und Seifert 2013).

Zusammenfassend lassen sich folgende (teilprojektübergreifende) Ziele mit Blick auf die Akteursgruppe der Studierenden formulieren (Niesyto 2019a, $24 \mathrm{f}$.):

- die Förderung eines Verständnisses von Medienbildung und Medienkompetenz, welches an den vorhandenen lebens- und medienweltlichen Erfahrungen der Schülerinnen und Schüler und dem vorhandenen $\mathrm{Me}$ dienwissen der Studierenden anknüpft;

- die Aneignung technischer und gestalterischer Kompetenzen für die Förderung von Selbstausdruck, Kommunikation und Lernen mit digitalen Medien in Grundschulkontexten;

- die gezielte Förderung visueller, auditiver und audiovisueller Ausdrucksformen in der produktiven Gestaltung mit digitalen Medien;

- die Aneignung informatischer Grundkompetenzen, um es Kindern in spielerischer Form zu ermöglichen, erste Elemente zu computerisierten Prozessen kennenzulernen;

- die Förderung reflexiver Prozesse bei der Produktion und Präsentation mit digitalen Medien;

- das Kennenlernen von Chancen des interdisziplinären Arbeitens.

Im Projekt waren die Abteilung Medienpädagogik und die Grundschulpädagogik beteiligt. Seitens der Fächer wirkten die Abteilungen Biologie, Deutsch, Englisch, Musik und Sport sowie das Institut für Mathematik und Informatik mit. Insgesamt gab es acht Teilprojekte. Einige Teilprojekte waren primär interdisziplinär, andere Teilprojekte primär fachdidaktisch ausgerichtet; ein Teilprojekt erprobte im Rahmen des Ganztagesangebots 
handlungsorientierte Kleinprojekte, auch in Kooperation mit ausserschulischen Partnern (Übersicht zu den Teilprojekten: siehe www.dileg-sl.de).

Ausgangspunkt und Grundlage waren Hochschulseminare (Begleitseminare), in denen insgesamt über 200 Studierende ca. 50 Unterrichtseinheiten für eine produktive Nutzung digitaler Medien erarbeiteten. In den Hochschulseminaren gab es in der Regel eine Tandembetreuung durch Personen aus der Medienpädagogik und den Fachdidaktiken. In jedem Teilprojekt fand zunächst eine Pilotphase statt, gefolgt (in der Regel im darauf folgenden Semester) von einer Durchführungsphase (jeweils zwei Semesterwochenstunden). Die Studierenden erprobten die von ihnen erarbeiteten Unterrichtseinheiten in Form von Unterrichtsversuchen in Grundschulklassen der Rosensteinschule in Stuttgart (Partnerschule) in Kooperation mit Lehrpersonen und werteten anschliessend ihre Erfahrungen im Hochschulseminar aus.

Die Rosensteinschule liegt in einem multikulturell geprägten Bezirk der Stuttgarter Innenstadt (Nordbahnhofviertel) und ist eine Grund- und Werkrealschule (teilgebundene Ganztagsschule). Die Grundschule besuchen etwa 200 Schülerinnen und Schüler verteilt auf 2- bis 3-zügige Klassenstufen. Der Anteil der Schülerinnen und Schüler mit Migrationshintergrund liegt bei über 90 Prozent. Am Projekt dileg-SL beteiligten sich insgesamt 185 Schülerinnen und Schüler (96 Jungen und 86 Mädchen; drei Kinder machten im Schülerfragebogen keine Angaben). ${ }^{2}$ Das Projekt entwickelte ein schulnahes Angebot für die Weiterbildung von Lehrpersonen und initiierte einen regionalen Arbeitskreis «Medienbildung in der Grundschule». ${ }^{3}$

Auf dem Hintergrund positiver Erfahrungen beim pädagogischen Einsatz von Tablets (u.a. Aufenanger 2015; Junge und Rust 2015; Schaal und Lude 2015; Trüby 2016), entschied sich die Projektgruppe, als technische

2 Webseite der Rosensteinschule: http://www.rosensteinschule.de/index. php?id=576. Weitere Informationen zu den Schülerinnen und Schülern sowie zu den Studierenden, die sich am Projekt dileg-SL beteiligten, siehe Niesyto 2019b, $207 \mathrm{f}$.

3 Informationen zum regionalen Arbeitskreis «Medienbildung in der Grundschule» siehe www.dileg-SL.de 
Basis für das Projekt vor allem Tablets zu nutzen. ${ }^{4}$ Das Mobile Device Management für die Schule erfolgte durch das Stadtmedienzentrum Stuttgart. Das Projekt präferierte einen mobilen Medieneinsatz im Sinne eines offenen Lernraums, der auch für andere Lernaktivitäten des Regelschulbetriebs geeignet ist (siehe Boelmann et al. 2019a). Für den Datenschutz entwickelte das Projekt ein detailliertes Konzept, welches nicht nur Einverständniserklärungen sondern auch ein Verfahrensverzeichnis umfasste, in dem Prozesse von der Erhebung, Verwendung bis hin zur Speicherung und Löschung der Daten dokumentiert und offengelegt wurden (Rymeš und Iberer 2019). ${ }^{5}$

\section{Wichtige Beobachtungen und Erfahrungen in teil- projektübergreifender Perspektive}

Da wir dileg-SL als ein praxisbezogenes Entwicklungsprojekt verstanden haben und aus dem Projektverlauf verschiedene Erkenntnisse gewinnen wollten, haben wir während des gesamten Projektzeitraums mehrere Massnahmen im Rahmen einer projektinternen, formativen Evaluation durchgeführt. Die Projektgruppe verständigte sich zu Beginn auf dem Hintergrund der skizzierten Projektziele auf teilprojektübergreifende Leitfragen der Evaluation (Niesyto 2019a, 32). Im Hinblick auf die Akteursgruppe der Studierenden standen folgende Leitfragen im Vordergrund:

- Welche Kompetenzen bzgl. digitaler Medien werden in den Begleitseminaren und den Unterrichtsversuchen bei den Studierenden sichtbar?

- Welche Erfahrungen machen Studierende und Dozierende im Projektverlauf? Inwieweit gelingt die Umsetzung der geplanten Unterrichtsversuche und Teilprojekte?

4 Nähere Informationen zur technischen Infrastruktur im Projekt dileg-SL und den Erfahrungen siehe Rymeš, Koppenhöfer, und Reichel 2019. Der Beitrag betont die Notwendigkeit verlässlicher technischer Infrastrukturen und von qualifiziertem Fachpersonal - an Grundschulen gibt es in diesem Bereich grosse Defizite und Herausforderungen (siehe auch Thumel und Metzler 2018).

5 Zum Projektende erschien eine Broschüre zum Thema «Datenschutz beim Einsatz digitaler Medien in der Grundschule» für Lehrpersonen an Grundschulen und Studierende (Rymeš, Walter, und Iberer 2019), die auch online verfügbar ist: https://www.ph-ludwigsburg.de/20840+M51093461ac2.html. 
Bei der projektinternen, formativen Evaluation wurden verschiedene Methoden eingesetzt (Niesyto 2019a, $32 \mathrm{f}$ ):

- eine Unterrichtsvideografie in zwei Teilprojekten in Verbindung mit speziellen Seminaren für Studierende; hier lag der Fokus auf der (quantitativen) Feinanalyse ausgewählter Schüler-Studierenden-Interaktionen (vgl. Kürzinger und Pohlmann-Rother 2019);

- Einzelinterviews mit den Dozierenden in den Teilprojekt-Teams zu wichtigen Erfahrungswerten (zu Beginn und nach der Pilotphase sowie am Ende der Durchführungsphase) sowie mit einzelnen Lehrkräften an der Schule;

- Projektjournale, die die beteiligten Studierenden in nahezu allen Begleitseminaren erstellten (Darstellung und Reflexion von Aktivitäten);

- Erhebung soziodemografischer und medienbezogener Informationen anhand von Fragebögen für Schülerinnen und Schüler;

- Hinzu kamen einzelne weitere Methoden, die die Teilprojekte entwickelten und einsetzten, z.B. Vignetten oder Videotagebücher.

Aufgrund personeller Veränderungen im Projektzeitraum und begrenzter Personal-/Zeitressourcen erfolgte bei der Evaluation (im quantitativen und qualitativen Bereich) eine Konzentration auf ausgewählte Aktivitäten und Leitfragenaspekte. Bei der qualitativen Evaluation des Gesamtprojekts lag der Fokus vor allem auf den Erfahrungs- und Lernprozessen bei den Studierenden (vgl. Niesyto 2019b; Junge 2019). Die Auswertung beruht auf einer Globalanalyse (Memos zu wichtigen Passagen in Projektjournalen der Studierenden und den transkribierten Interviews mit den Dozierenden) und nicht auf einer sequentiellen Feinanalyse der Dokumente. ${ }^{6}$ Kernpunkte der Auswertung, die zuvor in der Projektgruppe diskutiert wurden, flossen in einen Beitrag zur Nachhaltigkeit des Projekts ein (Junge, Niesyto, und Rymeš 2019). Der folgende Teil akzentuiert in

6 Weitere Informationen zu den Evaluationsmethoden (Erhebung und Auswertung) finden sich in Niesyto 2019a sowie im Online-Anhang zum Sammelband von Junge und Niesyto (2019, siehe https://kopaed.de/dateien/Junge_1106_ df_Online-Anhang.pdf). Dileg-SL war primär kein Forschungsprojekt, sondern ein praxisbezogenes Entwicklungsprojekt. Forschungsbezogene Vertiefungen gab es vor allem im Rahmen der Studien zur Unterrichtsvideografie (Kürzinger und Pohlmann-Rother 2019) und in den Teilprojekten 1 (Boelmann et al. 2019), 4 (Schaal 2019) und 8 (Bachelorarbeit von Walter 2017). 
teilprojektübergreifender Perspektive Ergebnisse, die sich bei der Gesamtauswertung der Dokumente als besonders wichtig herauskristallisierten.

\subsection{Medienkompetenzen der Studierenden}

Da alle Gespräche mit den Dozierenden durch einen vorab erstellten Leitfaden strukturiert wurden, war trotz der jeweils individuellen Gesprächsführung eine angemessene Vergleichbarkeit gegeben. Dennoch zeigten sich in den Gesprächen unterschiedliche Schwerpunktsetzungen in den einzelnen Teilprojekten und es wurden nicht alle Aspekte in der gleichen Intensität thematisiert (vgl. ausführlich Junge 2019). Die Medienkompetenzen der Studierenden wurden in allen Gesprächen umfassend erörtert, sodass wir hierzu verschiedene Aussagen treffen können.

Insgesamt ergibt sich aus den Beschreibungen der Lehrenden ein heterogenes Bild bzgl. der mitwirkenden Studierenden. Es zeigten sich deutliche Differenzen hinsichtlich der Fähigkeiten bei der Bedienung der bereitgestellten Geräte und Anwendungen, der Kreativität beim Medieneinsatz sowie der allgemeinen didaktischen Fähigkeiten/Routine.

Was die Bedienkompetenzen angeht, waren die Vorkenntnisse und Vorerfahrungen der Studierenden unterschiedlich ausgeprägt, sodass vonseiten der Dozierenden eigentlich keine grundsätzlichen Basiskenntnisse vorausgesetzt werden konnten. Vielmehr war es auffällig, dass die Lehrenden häufig von fehlenden Kompetenzen berichteten. So war es für viele Studierende durch die Mitwirkung an dileg-SL eine neue Erfahrung, mit Tablets produktiv zu arbeiten und in der Vorbereitung onlinebasierte Tools zur Zusammenarbeit (z.B. GoogleDocs oder Padlet) zu verwenden. Und selbst jene, die bereits im Vorfeld in verschiedenen Kontexten Tablets genutzt hatten, waren nur selten in der Lage, komplexere Herausforderungen (z.B. Verbindungsprobleme zwischen iPad, Software und eigenem heimischen PC) eigenständig zu lösen.

Die Lehrenden waren mehrheitlich überrascht, dass nur wenige Studierende umfassende technische Vorkenntnisse aufweisen konnten. Insgesamt zeigten sich die Dozenten-Teams aber davon überzeugt, in den Begleitseminaren ihren Teilnehmenden die notwendigen Fähigkeiten vermittelt zu haben, damit sie die Geräte sowie verschiedene Apps im 
Unterrichtsversuch erfolgreich anwenden konnten. Dies ging allerdings damit einher, dass mehr Zeit als erwartet für die Erlernung der grundlegenden Bedienung der bereitgestellten Technik benötigt wurde. Und bei weitem nicht alle Studierenden waren in der Lage, sich innerhalb der recht kurzen Vorbereitungszeit eine derart ausgeprägte Bedienkompetenz anzueignen, dass sie den Kindern tatsächlich bei Problemen oder komplexeren Anwendungswünschen weiterhelfen konnten. Dies erwies sich bei den Unterrichtsversuchen zwar nicht als gravierendes Hindernis, ist aber trotzdem problematisch. In einzelnen Gruppen wurde auch eine Rollenverteilung vorgenommen, dergestalt dass lediglich ein Student als «technischer Ansprechpartner» auserkoren wurde, was ebenfalls nicht unseren Intentionen entspricht.

Die Fokussierung auf technische Fragestellungen kam auch darin zum Ausdruck, dass etliche Studierende den Wunsch hatten, noch genauer in die Grundlagen der Tablet-Bedienung eingeführt zu werden (bspw. Erstellung von Dokumenten oder Übertragen von Daten zwischen Laptop und Tablet). Hierdurch wurden bedauerlicherweise andere, wichtige Bereiche (insbesondere ästhetische Fragen) vernachlässigt.

Bei mehreren Studierenden konnten die Lehrenden im Hinblick auf den Medieneinsatz einen Mangel an Kreativität feststellen. So wurde teilweise eine Verengung auf die im Seminar vorgestellten Anwendungen wahrgenommen. Während einzelne Gruppen den Input der Lehrenden aufgegriffen und durch eigene kreative Ideen ergänzt haben, ist es anderen demnach nur ansatzweise gelungen, die Potenziale der digitalen Medien umfassend zu nutzen. Möglicherweise zeigte sich hier auch eine gewisse Scheu, mit den gewährten Freiheiten umzugehen. Denn die Lehrenden berichteten sowohl in den Interviews nach der Pilotphase als auch nach der Durchführungsphase von Studierenden, die mit den offerierten Freiräumen nicht in der gewünschten Weise umgegangen sind. Vielmehr zeigte sich eine starke Orientierung an den Dozierenden und der Wunsch nach einer konkreten Anleitung. Eigene Impulse waren in diesen Fällen entweder nicht vorhanden oder wurden zugunsten der Frage nach «richtig» oder «falsch» zurückgehalten.

Dies korrespondiert mit der Wahrnehmung, dass etliche Studierende bei den Unterrichtsversuchen eine Tendenz zum lehrerzentrierten, 
gesteuerten Unterricht zeigten. Obwohl insbesondere die Arbeit mit Tablets Möglichkeiten eröffnet, um Schülerinnen und Schüler frei und explorativ arbeiten zu lassen (vgl. Autenrieth et al. 2018), wurden sie von etlichen Studierendengruppen angeleitet als Lernwerkzeug verwendet.

Eine weitere Beobachtung ist in diesem Kontext von Bedeutung. Obwohl die meisten Studierenden die Arbeit mit den digitalen Medien (insbesondere den bereitgestellten Tablets) überaus positiv bewerteten und auch die Einsatzmöglichkeiten für den Fachunterricht in der Grundschule erkannt haben, war bei vielen Lehramtsstudierenden zum Beginn des Projekts eine skeptische Haltung bezüglich des Einsatzes digitaler $\mathrm{Me}$ dien in der Grundschule vorhanden (vgl. hierzu ähnliche Ergebnisse bei Brüggemann 2013) und sie zeigten deutliche Berührungsängste. Zudem berichteten die Lehrenden von vereinzelten Vermeidungstendenzen. So wurde bei einzelnen Arbeitsschritten von den Teilnehmenden wieder auf bekannte Software und den ihnen vertrauten analogen Arbeitsformen zurückgegriffen. Die digitalen Medien bzw. einzelne Anwendungen, die sie im Seminar kennengelernt hatten, wurden somit nur partiell eingesetzt.

Offen bleibt an dieser Stelle, ob diese Vermeidungstendenzen auf eine Unsicherheit hinsichtlich der Bedienung zurückzuführen sind oder ob es sich hierbei lediglich um Gewohnheiten handelt. Möglicherweise liegt es auch darin begründet, dass der Einsatz der digitalen Medien schon als unbekanntes Terrain erachtet wurde, weswegen ansonsten lieber «sichere Pfade» gewählt wurden.

Nicht unerwähnt bleiben darf, dass sich auf der anderen Seite einzelne Studierende bereits eine grosse Expertise im Bereich der digitalen Medien angeeignet hatten, die sie im Kontext von dileg-SL auch nutzbar gemacht haben. Diese Studierenden brachten sich mit grossem Engagement ein, benötigten keine umfassende technische Schulung mehr und brachten kreative Ideen bzgl. des Medieneinsatzes ein.

Insgesamt haben unsere Beobachtungen aber gezeigt, wie wichtig bei praxisorientierten Projekten wie dileg-SL die Vermittlung von technischen Bedienkompetenzen ist. Bei einem grossen Teil der Lehramtsstudierenden, die am Projekt dileg-SL mitgewirkt haben, zeigte sich im Kontext der Seminare und Unterrichtsversuche ein deutlicher Bedarf nach Aneignung technischer und gestalterischer Medienkompetenzen. Hierfür ist 
vonseiten der Lehrenden genügend Zeit einzuplanen, insbesondere wenn im Rahmen solcher Projekte zum ersten Mal eine intensive Auseinandersetzung mit den gestalterischen Möglichkeiten von Tablets stattfindet. Schliesslich benötigten etliche Studierende aufgrund ihrer geringen VorErfahrungen bezüglich der produktiven Gestaltung mit digitalen Medien mehr Zeit als andere Teilnehmende, um sich entsprechende Basiskompetenzen aneignen zu können. Aber ohne technisch-gestalterische und mediendidaktische Basiskompetenzen ist es nicht möglich, Medien zielführend mit fachdidaktischen Themen zu verknüpfen und pädagogisch-didaktisch situationsadäquat einzusetzen sowie sich in reflektierenden Prozessen damit auseinanderzusetzen, inwieweit digitale Medien auch zu einer veränderten Lernkultur beitragen können.

\subsection{Potenziale visueller und audiovisueller Digitalmedien}

Gerade aus grundschulpädagogischer Perspektive ist es wichtig, dass Kinder die Möglichkeit haben, sich die Welt aus unterschiedlichen Perspektiven und in unterschiedlichen Formen zu erschliessen. Die systematische Integration von visuellen und audiovisuellen Digitalmedien erwies sich im Projekt als geeignet, um Schülerinnen und Schüler in einem hohen Masse in kreative und kooperative Lernformen einzubinden und damit auch inklusive Zielsetzungen zu stärken. Sprach- und Schreibbarrieren konnten minimiert, Lerngegenstände und -inhalte anschaulich dargestellt und digitale Medien flexibel für Recherchen, zur Dokumentation vor Ort, zur Visualisierung und zur Kommunikation eingesetzt werden. Nahezu allen Kindern fiel es leicht, die iPads und die verschiedenen Apps für Foto- und Filmaufnahmen zu bedienen. So betonte das Team eines Teilprojekts (Bereich Deutsch und Medienpädagogik):

«Schülerinnen und Schüler, die ansonsten Schwierigkeiten hatten, ihre Gedanken sprachlich zu formulieren, nutzten die digitalen Lernumgebungen, um durch Deuten, Verschieben, Abspielen oder Vergrössern einzelner Sequenzen auf das für sie Zentrale hinzuweisen und so mit den anderen Kindern ins Gespräch zu kommen» (Boelmann, König, und Rymeš 2019b, 54). 
In einem anderen Teilprojekt (Bereich Musik und Medienpädagogik) wurde u.a. die Möglichkeit der schnellen und kleingliedrigen Folgen von Gestaltungs- und Korrekturschritten (bezüglich Bilder und Töne) bei den genutzten Apps GarageBand und StopMotionStudio hervorgehoben, insbesondere mit Blick auf jene Schülerinnen und Schüler, die im Unterricht oft Konzentrationsprobleme haben. Diese Möglichkeit förderte eigene ästhetische Erfahrungen als Basis für ästhetische Gestaltung (Imort und Trüby 2019, 84). Bei Unterrichtsversuchen im Fach Englisch erwiesen sich die integrierten Bilder und Aussprachebeispiele für alle Kinder als ein verständlicher, niedrigschwelliger Einstieg, auch für Kinder mit diagnostiziertem Förderbedarf. Das Team resümierte:

«Insbesondere gelang es den Studierenden, Schülerinnen und Schüler zu motivieren, leistungsschwächeren Kindern mehr Teilhabe im Unterricht zu ermöglichen und neue Möglichkeiten des sozialen Lernens zu implementieren» (Rymeš, Keßler, und Jokiaho 2019, 142).

Auf der Abschlusstagung des Projekts betonte eine Lehrerin der Rosensteinschule, dass es diverse Beispiele gab, wo Kinder mit einem speziellen Förderbedarf auf Augenhöhe mit anderen Kindern zusammengearbeitet hatten. Eine Projektmitarbeiterin ergänzte:

«In einzelnen Situationen wusste ich nicht, dass Kinder mit einem speziellen Förderbedarf am Angebot teilnahmen. Ich hatte es in der Praxis überhaupt nicht wahrnehmen können, erst im Nachhinein erfahren».

Diese positiven Erfahrungen mit der Integration von Foto und Bewegtbild in digitale Medienproduktionen (siehe vor allem Boelmann, König, und Rymeš 2019b; Imort und Trüby 2019; Autenrieth et al. 2018) bestätigen Ergebnisse aus früheren medienpädagogischen Praxisforschungsprojekten (Witzke 2004; Niesyto et al. 2007) und aus deutschen und britischen Schulprojekten auf der Basis multimodaler Lerndesigns (Bachmair 2019).

Insgesamt verdeutlichten die Projekterfahrungen das Interesse der Studierenden, praxisbezogen digitale Gestaltungsmöglichkeiten durch die Integration von Text, Ton, Foto und Bewegtbild kennenzulernen. Zugleich zeigten Beobachtungen in verschiedenen Seminar- und 
Unterrichtssituationen, dass erheblich mehr Anstrengungen und Zeit zur Förderung von Bild- und Filmkompetenz notwendig sind. Gerade in einer Situation, in der bildungspolitische Programme gebetsmühlenartig die «Digitalisierung» beschwören, wird oft übersehen, dass Visualität in der digitalen Welt nicht abgenommen, sondern weiter zugenommen hat (Reißmann 2015). So wünschten sich z.B. die Befragten in einer repräsentativen Studie zur YouTube-Nutzung junger Menschen explizit mehr Anregungen zur Filmgestaltung in der Schule (vgl. Rat für kulturelle Bildung 2019, 34).

Grundlegende ästhetische Merkmale analoger Medien sind auch in digitalen Formen relevant. Es ist eine Bildungsaufgabe, digitale Ästhetiken auch unter Rückbezug auf analoge Ästhetiken zu reflektieren. So gibt es zum einen bei digitalen Medien (und dazugehöriger Software) z.B. grosse Potenziale zur Bildbearbeitung, die zuvor im analogen Bereich nicht möglich waren. Andererseits offerieren aber viele Apps auch gestalterische Standardisierungen, die zwar für Erstlingsproduktionen niedrigschwellige Zugänge ermöglichen, ästhetische Gestaltungsprozesse aber auch begrenzen. Diese Fragen sollten Bestandteil von Reflexionen bei Produktionsprozessen sein.

\subsection{Reflexive Prozesse bei Projektaktivitäten}

In medienbezogenen Aktivitäten wird zurecht insbesondere auf die Förderung von gestalterischen Kompetenzen beim aktiven Umgang mit digitalen Medien hingewiesen. Es ist allerdings immer wieder zu beobachten, dass Formen der Reflexion bei der aktiven Nutzung digitaler Medien zu kurz kommen. Was waren die Beobachtungen und Erfahrungen im Projekt dileg-SL hierzu?

Dokumenten aus den Teilprojekten ist zu entnehmen, dass in den Begleitseminaren an der Hochschule Reflexionen vor allem bei der kooperativen Planung, nach der Präsentation von Medienproduktionen und bei der Auswertung der Unterrichtsversuche stattfanden (thematische, sozial-kommunikative, ästhetische und technische Aspekte). Es gab auch Teilprojekte, die kontinuierlich reflexive Feedbackrunden integrierten (Studierende mit Beteiligung von Dozierenden), vor allem durch eine enge 
Verzahnung von Vorbereitung, Durchführung und Reflexion von Unterricht. Insgesamt verdeutlichte die Projektpraxis, dass reflexive Phasen während des Produktionsprozesses oft zu kurz kamen, um aus dem Modus der Produktion heraus über die selbst erstellten Medienprodukte nachzudenken, z.B. Impulse zur Auseinandersetzung mit der Qualität der eigenen Materialien und Hinweise auf gestalterische Überarbeitungs- und Verbesserungsmöglichkeiten. Hier geht es um (produktionsbegleitende) kognitive Aktivierungen, um anschauliches Denken und ästhetische Reflexionen. Diese ästhetischen Prozesse und Reflexionen benötigen auch Zeit für intuitiv-tastende, streunende, experimentelle Suchbewegungen.

Mit dem eigenen Verständnis von Kindheit, Bildung, Lernen, Medien setzten sich die Studierenden in den Hochschulseminaren des Projekts in unterschiedlicher Intensität auseinander. Die Projekterfahrungen zeigten: Digitale Medienproduktionen haben Potenziale für Formen selbstgesteuerten Lernens auch an Grundschulen, wenn genügend Zeit vorhanden ist, mit Studierenden ihr Rollen- und Selbstverständnis als Lehrperson zu reflektieren. Die im Projekt beobachtete Unterrichtspraxis verdeutlichte (wie bereits oben angedeutet), dass bei vielen Studierenden (teilweise auch aus höheren Semestern) oft noch relativ starre und strukturkonservative Vorstellungen von Unterrichtsplanung und -gestaltung existieren, die Schülerinnen und Schüler mehr als «Aufgabenempfänger» und weniger als Ko-Konstrukteure ihrer eigenen Lernprozesse betrachten. Dabei ist zu beachten, dass Studierende nur begrenzt eigene Unterrichtserfahrungen (aus der Perspektive von Lehrpersonen) haben.

Lehrerbildung hat hier die Aufgabe, für Studierende ausreichend Reflexionsräume zu schaffen und sie dafür zu sensibilisieren, vorhandene Deutungs- und Orientierungsmuster zu hinterfragen und eigenes, medienbezogenes Erfahrungslernen zu wagen. Wie sehen Studierende das Spannungsfeld von eher sachbezogenen Funktionslogiken einerseits und Formen der Weltaneignung von Kindern andererseits? Wie wird bei Medienproduktionen das Spannungsfeld zwischen Prozess- und Produktorientierung situativ gesehen und gestaltet? Welche Möglichkeiten gibt es in diesem Zusammenhang, um Grundschulkindern altersgemässe Artikulationsmöglichkeiten und Freiräume zu eröffnen, die sie weder unter- noch überfordern? Wie kann dies durch die Berücksichtigung der jeweiligen 
Vorerfahrungen der Kinder und durch unterschiedlich stark strukturierte Aufgabenstellungen und Lernumgebungen - auch ausserhalb des Klassenzimmers - erreicht werden? Wie können reflexive Phasen aus dem Modus der Gestaltung/Produktion mit digitalen Medien heraus kleinschrittig entwickelt werden?

Die Auseinandersetzung mit diesen (und weiteren) Fragen umfasst auch den Austausch und die kritische Reflexion zwischen den beteiligten Hochschullehrenden: Was für Verständnisse von Kindheit, Bildung, Lernen, Medien existieren unter Dozierenden? Wie können unterschiedliche konzeptionelle Akzentuierungen zu einem produktiven Faktor für den kollegialen Austausch, für die Lehre und die Studierenden gemacht werden? Das Projekt dileg-SL eröffnete hierfür Räume, die von den Teilprojekten unterschiedlich genutzt wurden. Die Zusammenarbeit in interdisziplinären Teams war vor allem dann spannend, wenn die Dozierenden bereit waren, ihre jeweiligen Expertisen kritisch-konstruktiv in die Kooperation einzubringen und Studierenden die Chance gaben, verschiedene Ansätze und Überlegungen zu Bildungs- und Lernprozessen kennenzulernen und zu reflektieren. Dabei erwies es sich als notwendig, die thematische Komplexität zu reduzieren, damit Studierende in einem ausgewogenen Verhältnis fachdidaktische, medien- und grundschulpädagogische Überlegungen mit medienpraktischen Erkundungen und der Planung, Durchführung und Auswertung exemplarischer Unterrichtsversuche verbinden konnten. ${ }^{7}$

\section{Fazit: Grundbildung Medien ist mehr als digitale Grundbildung}

Die Initiative «Keine Bildung ohne Medien!» und die Sektion Medienpädagogik in der DGfE verweisen in ihren Forderungen und Positionspapieren schon seit vielen Jahren auf die Notwendigkeit einer Grundbildung Medien für alle Studierenden in der Primarstufenbildung. An der PH Ludwigsburg wird dies bereits seit 2012 umgesetzt (Trüby 2017) und im Projekt «Digitales Lernen Grundschule» durch die Tandem-Betreuung (Kooperation $\mathrm{Me}$ dienpädagogik mit Fachdidaktiken) erfolgte in den zahlreichen Seminaren

7 Siehe hierzu die curricularen und hochschuldidaktischen Überlegungen in Junge, Niesyto, und Rymeš 2019. 
eine inhaltliche Vertiefung. Die Forderung nach einer Grundbildung Medien knüpft dabei an der Fächerperspektive an, geht aber darüber hinaus. Sie umfasst weit mehr als eine «Digitale Grundbildung» - dies zeigen die diversen Erfahrungen des Projekts dileg-SL.

Letztendlich können wir festhalten, dass Projekte wie dileg-SL einen wichtigen Beitrag leisten können, um Lehramtsstudierende mit den Potenzialen digitaler Medien vertraut zu machen. Neben der Vermittlung von Fähigkeiten im Umgang mit den technischen Geräten geht es aber auch darum, die Einstellungen der Lehramtsstudierenden in den Blick zu nehmen.

So waren zwar die meisten Studierenden, die in den Teilprojekten von dileg-SL mitgewirkt haben, neugierig und bereit, sich auf Neues einzulassen. Aber obwohl der Medienbezug in der Seminarausschreibung deutlich benannt wurde, zeichneten sich keineswegs alle Studierenden durch eine hohe Medienaffinität und einen sicheren Umgang mit den digitalen Medien aus (siehe Kapitel 3.1). Dies bestätigt die Zweifel an dem mitunter kolportierten Klischee der digitale natives (vgl. u.a. Ganguin und Meister 2013; Schulmeister und Loviscach 2017). Die Auseinandersetzung mit den digitalen Medien im Kontext der Grundschule wurde von einzelnen Lehramtsstudierenden sogar als notwendiges Übel betrachtet. Dabei ist es wichtig, dass Prozesse der Digitalisierung auch kritisch reflektiert werden und es sollte keineswegs darum gehen, die fortschreitende Etablierung digitaler Medien ausschliesslich euphorisch zu betrachten. In der Vergangenheit wurden die Potenziale «neuer» Medien oftmals überschätzt. Gleichzeitig ist es problematisch, wenn eine grundsätzlich skeptische Haltung und eine unreflektierte Distanz gegenüber digitalen Medien dafür sorgt, dass angehende Lehrkräfte die unbestreitbar vorhandenen Potenziale für die Unterrichtsgestaltung nicht nutzbar machen (können).

Neben einer positiven Einstellung gegenüber digitalen Medien und einer Neugier gegenüber den neuen Möglichkeiten ist es erforderlich, dass (angehende) Lehrpersonen über eigene Medienkompetenz und über medienpädagogische, mediendidaktische und technisch-gestalterische Kompetenzen verfügen. Nur so können digitale Medien in Verbindung mit verschiedenen Themen pädagogisch sinnvoll und zielführend im schulischen Alltag sowie im Fachunterricht Anwendung finden. Wie bereits dargelegt 
mussten wir im Rahmen unseres Projekts feststellen, dass zu viele Studierende nicht über hinreichende Kompetenzen verfügen, die über den alltäglichen Gebrauch von Smartphones und Computern hinausgehen. Um dieses Defizit auszugleichen sollten die ausbildenden Hochschulen in verstärktem Masse entsprechende Angebote machen.

Vor dem Hintergrund unserer Projekterfahrungen planen verschiedene am Projekt beteiligte Dozierende die Fortsetzung interdisziplinärer Seminare mit Medienbezug und es wurde das Konzept für ein medienpädagogisches Basisseminar «Medienbildung und Lernen mit digitalen Medien in der Grundschule» entwickelt. In diesem Kurs sollen die Studierenden für die vielfältigen medialen Erfahrungen von Kindern sensibilisiert werden und ihnen werden die Potentiale digitaler Medien für den Einsatz im Unterricht vermittelt. Dabei ist auch zukünftig ein weites Verständnis von Medien und Medienbildung notwendig, um den Herausforderungen angemessen begegnen zu können. Hierzu gehören für uns

- umfassende Grundkenntnisse zur Mediensozialisation von Kindern und Jugendlichen

- eine vertiefte Auseinandersetzung mit dem Verständnis von Kindheit, Bildung, Lernen und Medien

- und Grundverständnis für eine altersgemässe, inklusive und nicht zuletzt milieusensible Medienbildung

- Grundkenntnisse zu verschiedenen «Mediensprachen» (vor allem mit Blick auf ästhetisch-gestalterische Potenziale)

- Grundkenntnisse zu algorithmischen Prozessen und Computational Thinking

- Befähigung zu einer differenzierten Medienkritik, zur Reflexion problematischer gesellschaftlicher Medienentwicklungen, zu medienethischen Aspekten, zur Auseinandersetzung mit den jeweils aktuellen Fragen des Datenschutzes

- genügend Zeit für das eigenständige und/oder angeleitete Erproben ästhetischer und technischer Gestaltungsmöglichkeiten digitaler $\mathrm{Me}$ dien.

Hinsichtlich der Platzierung solcher Seminarangebote wäre es wichtig, entsprechende Seminare noch stärker als bisher verpflichtend in den Studienordnungen zu verankern. Hierfür wären Vorgaben vonseiten des 
Ministeriums hilfreich, die eine Grundbildung Medien für alle Lehramtsstudierende verbindlich machen. Schliesslich ist kaum zu erwarten, dass die junge Lehrergeneration nach erfolgreichem Berufsstart dazu fähig ist, die Potenziale der digitalen Medien in ihrer Arbeit als Lehrerin und Lehrer nutzbar zu machen, wenn sie während des gesamten Studiums gar nicht oder nur sehr vereinzelt in Berührung gekommen sind. Die aktuellen Diskussionen zeigen, dass schon bei der aktuellen Lehrergeneration in diesem Bereich ein grosser Weiterbildungsbedarf besteht, der im Zusammenhang mit dem Digitalpakt noch zunehmen wird.

Das «Profil Grundbildung Medien», wie wir es an der PH Ludwigsburg seit einigen Jahren erfolgreich anbieten, stellt einen geeigneten curricularen Rahmen dar, der für alle Lehramtsstudierende verpflichtend gemacht werden sollte. Das «Profil Grundbildung Medien» setzt an vorhandenen Angeboten der Bildungswissenschaften, der Fachdidaktiken und des $\mathrm{Me}$ dienzentrums an, führt in einem Modul vier Bausteine kompakt zusammen und berücksichtigt mit einem zu realisierenden Medienprojekt auch die Perspektive des praktischen und produktionsorientierten Bezugs zu (digitalen) Medien. Nicht zuletzt für die Umsetzung eines selbst entwickelten Medienprojekts benötigen wir an den Hochschulen spezifische Ressourcen, die im Hinblick auf eine Grundbildung Medien für alle Studierenden mit den aktuell zur Verfügung stehenden Personal- und Sachmitteln kaum zu leisten sind. Hierfür müssten entsprechende Mittel dauerhaft zur Verfügung gestellt werden.

Die dauerhafte Einrichtung entsprechender Ressourcen lässt sich nicht nur damit begründen, dass die enormen medialen Herausforderungen eine «Grundbildung Medien» notwendig machen, die im späteren Beruf durch medienbezogene Fort- und Weiterbildungen zu aktualisieren ist. Darüber hinaus ist die Qualifizierung aller Lehrkräfte auch als Beitrag zur Bildungsgerechtigkeit zu betrachten. Schon in der 1. Phase der Lehrerbildung wird die Basis dafür gelegt, dass angehende Lehrkräfte an allen Grundschulen über hinreichende Medienkompetenzen und medienpädagogische Kompetenzen verfügen, um einen guten Unterricht mit und über digitale Medien machen zu können. Diese Einschätzung korrespondiert mit der Stellungnahme des Grundschulverbandes «Digitale Mündigkeit beginnt in der Grundschule» vom April 2018. 


\section{Schlussfolgerungen und kritischer Ausblick}

Eine Grundbildung Medien in der Primarstufenbildung, wie sie im vorigen Teil dieses Beitrags skizziert wurde, arbeitet mit einem breiten $\mathrm{Me}$ dienbegriff und einem umfassenden Verständnis von Medienbildung. Es geht nicht nur um digitale, sondern auch um analoge Medien und es wird ein Verständnis von Medialität und Digitalität zugrunde gelegt, welches symbolisch-sinnhafte und kulturell-kommunikative Dimensionen betont (u.a. Stalder 2016). Gerade aus grundschulpädagogischer Perspektive ist das Zusammenspiel unterschiedlicher Erfahrungsmodi von Welt zu betonen. Digitale Technik und digitale Ästhetik verändern zwar bisherige analoge Zeichen- und Symbolsysteme und es ist wichtig, dass sich Studierende digitalitätsbezogene Kompetenzen aneignen und die Funktionen und Spezifika digitaler Informationsverarbeitung verstehen, bewerten und z.B. in MakerSpace-Projekten Erfahrungen sammeln (Ingold et al. 2019). Digitale Technik und digitale Ästhetik führen jedoch nicht generell zum Verschwinden analoger Zeichen- und Symbolsysteme und damit verbundenen Erfahrungen. In digital- und medienkritischer Perspektive ist zu formulieren: «Nur im Kontext von nicht-digitalen Erfahrungen lässt sich erkennen, was digital begrenzt wird bzw. digital nicht oder noch nicht möglich ist» (Kulcke 2018, 188).

Auf dem Hintergrund dieser Schlussfolgerungen, die wir in Zusammenhang mit dem Projekt dileg-SL hervorheben möchten, sollen abschliessend einige kritische Überlegungen mit Blick auf aktuelle Entwicklungstrends skizziert werden. So übersieht der gegenwärtige Digital-Hype, dass es neben Wandel und «Disruption» auch Kontinuitäten gibt. Die Bezeichnung «Digitale Bildung» ist nicht nur sachlich falsch (vgl. Vollbrecht 2018, 26) und ein «Label, das für alles und nichts gebraucht werden kann» (Kübler $2018,17)$, sondern verkürzt vor allem den Blick auf die Vieldimensionalität der pädagogischen Aufgabenstellung. Immer mehr an den Rand geraten grundlegende Fähigkeiten, die für Bildungs- und Lernprozesse elementar sind, wie z.B. Reflexions- und Kritikfähigkeit, bildungs- und lerntheoretische Grundlagen und elementare pädagogisch-didaktische und medienpädagogische Kompetenzen.

Auch ist darauf hinzuweisen, dass der Einfluss der (Internet-) Wirtschaft auf die schulische Bildung immer grösser wird. Während 
Wissenschaftsministerien und die meisten Hochschulen sich nicht in der Lage sehen, eine umfassende Medienbildung breitenwirksam und verbindlich in pädagogischen Studiengängen zu verankern (Knaus, Meister, und Tulodziecki 2018, 30 f.), bieten diverse Firmen Schulen kostenlose Workshops für Lehrkräfte und kostenlose Unterrichtsmaterialien an. Über verschiedene Werbemassnahmen gibt es inzwischen subtile Formen der Beeinflussung. Auch ist eine zunehmende Verflechtung von Bildungspolitik und Digitalwirtschaft zu beobachten (vgl. Schmerr 2019). Zugleich gibt es bis dato keine massentauglichen Alternativen in Deutschland und Europa im Bereich der Hard- und Software zu den marktbeherrschenden Angeboten der grossen Internetkonzerne (Staab 2019), die für Bildungseinrichtungen geeignet wären und nicht mit der ständigen Enteignung von Daten verbunden sind. So erfreulich die finanzielle Unterstützung der Schulen durch den Digitalpakt auch ist und so dringend erforderlich eine grundlegende technische Infrastruktur auch sein mag, darf dies in den aktuellen Debatten nicht übersehen werden!

In dieser Situation ist es wichtig, dass schulische Bildung und auch Lehre und Forschung an Hochschulen ihre Unabhängigkeit von kommerziellen Interessen bewahren und dem gegenwärtigen Mainstream einer Digitalisierung der Pädagogik widerstehen. Dies bedeutet auch, Ziele und Strukturen einer technologie- und profitgetriebenen Digitalisierung kritisch zu hinterfragen (Niesyto 2018, 54 ff.; Hug 2018, 9 ff.) und gleichzeitig Schule zu transformieren: erheblich mehr Gestaltungsräume für die Schülerinnen und Schüler, mehr Interdisziplinarität und weniger Fixierung auf Fächer, flexiblere Raum- und Zeitstrukturen und kompetente Lehrpersonen, die ihre Rolle mehr als Lernbegleitende verstehen und in der Lage sind, mit Schülerinnen und Schüler offene Medienbildungsräume zu erschliessen (Schiefner-Rohs 2017). 


\section{Literatur}

Aufenanger, Stefan. 2015. «Tablets an Schulen. Ein empirischer Einblick aus der Perspektive von Schülerinnen und Schülern.» In Smart und mobil: digitale Kommunikation als Herausforderung für Bildung, Pädagogik und Politik, herausgegeben von Katja Friedrich, 63-77. München: kopaed.

Autenrieth, Daniel, Anja Marquardt, Horst Niesyto, und Katrin Schlör. 2018. «Digitales Lernen in der Grundschule - ein Werkstattbericht mit Praxisbeispielen aus dem Projekt dileg -SL». In Spannungen und Potenziale. Digitaler Wandel in Bildungseinrichtungen, herausgegeben von Thomas Knaus und Olga Engel, 151-174. München: kopaed.

Bachmair, Ben. 2019. «Exploring Writing in a Digitally-Dominated Culture Options for Formal Learning in Schools». Media Education Nr. 3 (2019), 338366. http://ejournal53.com/journals_n/1568979994.pdf und https://doi.org/ 10.13187/me.2019.3.338.

Boelmann, Jan M., Lisa König, und Robert Rymeš. 2019a. «Actioncams und Kameraroboter. Ein mobiles Konzept zur technischen Umsetzung von Unterrichtsvideografie». In Digitale Medien in der Grundschullehrerbildung. Erfahrungen aus dem Projekt dileg-SL, herausgegeben von Thorsten Junge und Horst Niesyto, 195-203. München: kopaed.

Boelmann, Jan M., Lisa König, und Robert Rymeš. 2019b. «Vom Storyboard zum eigenen Film. Schul- und Hochschulpädagogische Erfahrungen aus dem Teilprojekt ,Digital Storytelling und intermediales Geschichtenverstehen'». In Digitale Medien in der Grundschullehrerbildung. Erfahrungen aus dem Projekt dilegSL, herausgegeben von Thorsten Junge und Horst Niesyto, 43-56. München: kopaed.

Breiter, Andreas, Stefan Aufenanger, Ines Averbeck, Stefan Welling, und Marc Wedjelek. 2013. Medienintegration in der Grundschule. Untersuchung zur Förderung von Medienkompetenz und der unterrichtlichen Mediennutzung in Grundschulen sowie ihrer Rahmenbedingungen in Nordrhein-Westfalen. Schriftenreihe Medienforschung der LfM (Band 73). Berlin: Vistas. http://www.lfm-nrw.de/ fileadmin/lfm-nrw/Forschung/Kurzfassung_Studie_73.pdf.

Brüggemann, Marion. 2013. Digitale Medien im Schulalltag. Eine qualitativ rekonstruktive Studie zum Medienhandeln und berufsbezogenen Orientierungen von Lehrkräften. München: kopaed.

Deckert-Peaceman, Heike, und Anja Seifert. 2013. «Die Grundschule als Ort grundlegender Bildung?» In Die Grundschule als Ort grundlegender Bildung Beiträge zu einer Neuverortung der Grundschulpädagogik, herausgegeben von Heide Deckert-Peaceman und Anja Seifert, 7-20. Bad Heilbrunn: Klinkhardt.

Deutsche Telekom Stiftung, Hrsg. 2014. Medienbildung entlang der Bildungskette. Bonn. https://www.telekom-stiftung.de/sites/default/files/files/media/publications/buch_medienbildung.bildungskette_end.pdf.

Ganguin, Sonja, und Dorothee Meister, Hrsg. 2013. Digital native oder digital naiv? - Medienpädagogik der Generationen. München: kopaed. 
Grundschulverband. 2018. «Digitale Mündigkeit beginnt in der Grundschule». https://grundschulverband.de/wp-content/uploads/2018/08/stellungnahmegsv-digitalpakt-Schule.pdf.

Herzig, Bardo. 2014. Wie wirksam sind digitale Medien im Unterricht? Studie im Auftrag der Bertelsmann Stiftung. Gütersloh: Bertelsmann Stiftung. https://www. bertelsmann-stiftung.de/fileadmin/files/BSt/Publikationen/GrauePublikationen/Studie_IB_Wirksamkeit_digitale_Medien_im_Unterricht_2014.pdf.

Hug, Theo. 2018. «Herausforderungen für Lernen und Bildung im Medienzeitalter - Zur Einführung». In Medienpädagogik. Herausforderungen für Lernen und Bildung im Medienzeitalter, herausgegeben von Theo Hug, 7-17. Innsbruck: innsbruck university press.

Imort, Peter, und Horst Niesyto, Hrsg. 2014. Grundbildung Medien in pädagogischen Studiengängen. Schriftenreihe Medienpädagogik interdisziplinär, Band 10. München: kopaed.

Imort, Peter, und Daniel Trüby. 2019. «Zwischen Märchenwesen und Wasserkreislauf. Gestaltungsorientierte Trickfilmproduktionen mit Drittklässlern. Aspekte eines interdisziplinären Projekts der Medien- und Musikpädagogik». In Digitale Medien in der Grundschullehrerbildung. Erfahrungen aus dem Projekt dileg-SL, herausgegeben von Thorsten Junge und Horst Niesyto, 73-85. München: kopaed.

Ingold, Selina, Björn Maurer, Daniel Trüby, Hrsg. 2019. Chance Makerspace. Making trifft auf Schule. München: kopaed.

Irion, Thomas, und Rudolf Kammerl. 2018. «Mit digitalen Medien lernen. Grundlagen, Potenziale und Herausforderungen». Die Grundschulzeitschrift 307, 12-17.

Junge, Thorsten. 2019. «Ergebnisse des Entwicklungsprojekts dileg-SL. Eindrücke und Befunde aus den begleitenden Befragungen der Dozenten-Teams». In Digitale Medien in der Grundschullehrerbildung. Erfahrungen aus dem Projekt dilegSL, herausgegeben von Thorsten Junge und Horst Niesyto, 233-262. München: kopaed.

Junge, Thorsten, und Horst Niesyto, Hrsg. 2019. Digitale Medien in der Grundschullehrerbildung. Erfahrungen aus dem Projekt dileg-SL. Schriftenreihe Medienpädagogik interdisziplinär, Band 12. München: kopaed.

Junge, Thorsten, Horst Niesyto, und Robert Rymeš. 2019. «Überlegungen zur Nachhaltigkeit des Entwicklungsprojekts dileg-SL». In Digitale Medien in der Grundschullehrerbildung. Erfahrungen aus dem Projekt dileg-SL, herausgegeben von Thorsten Junge und Horst Niesyto, 319-344. München: kopaed.

Junge, Thorsten, und Christiane Rust. 2015. «Lehrkräfte in der digitalen Welt. Einsatz von Tablet-PCs zur Entwicklung praxisorientierter Konzepte zur Prävention von Cybermobbing». In Lehrer.Bildung.Medien - Herausforderungen für die Entwicklung von Schule(n), herausgegeben von Mandy Schiefner-Rohs, Claudia Gomez, Christine Menzer, 171-184. Baltmannsweiler: Schneider-Verlag Hohengehren. 
KBoM - Initiative «Keine Bildung ohne Medien!» 2011. Bildungspolitische Forderungen. Medienpädagogischer Kongress 2011. http://www.keine-bildungohne-medien.de/kongress-dokumentation/keine-bildung-ohne-medien_bildungspolitische-forderungen.pdf.

KMK - Kultusministerkonferenz. 2016. Bildung in der digitalen Welt. Strategie der Kultusministerkonferenz. https://www.kmk.org/fileadmin/Dateien/pdf/PresseUndAktuelles/2017/Strategie_neu_2017_datum_1.pdf.

KMK - Kultusministerkonferenz. 2012. Medienbildung in der Schule. http:// www.kmk.org/fileadmin/Dateien/veroeffentlichungen_beschluesse/2012/2012_03_08_Medienbildung.pdf.

Knaus, Thomas, Dorothee M. Meister, und Gerhard Tulodziecki. 2018. «Qualitätsentwicklung - Professionalisierung - Standards. Thesen aus medienpädagogischer Sicht». In Futurelab Medienpädagogik. Qualitätsentwicklung - Professionalisierung - Standards, herausgegeben von Thomas Knaus, Dorothee M. Meister, Kristin Narr, 23-47. München: kopaed.

Kübler, Hans-Dieter. 2018. «Was ist und was soll digitale Bildung?» medien + erziehung, Heft 5 (2018), 16-24.

Kürzinger, Anja, und Sanna Pohlmann-Rother. 2019. «Die videogestützte Evaluation im Projekt dileg-SL. Leitfragen, Ergebnisse und Perspektiven für die Grundschullehrerbildung». In Digitale Medien in der Grundschullehrerbildung. Erfahrungen aus dem Projekt dileg-SL, herausgegeben von Thorsten Junge und Horst Niesyto, 263-276. München: kopaed.

Kulcke, Gesine. 2018. «Medienkritik in der Kindheitspädagogik im digitalen Zeitalter». In Medienkritik im digitalen Zeitalter, herausgegeben von Horst Niesyto und Heinz Moser, Schriftenreihe Medienpädagogik interdisziplinär, Band 11, 179-191. München: kopaed.

Kutscher, Nadja, und Hans-Uwe Otto. 2010. «Digitale Ungleichheit - Implikationen für die Betrachtung digitaler Jugendkulturen». In Digitale Jugendkulturen, herausgegeben von Kai-Uwe Hugger, 73-87. Wiesbaden: VS Verlag.

Niesyto, Horst. 2019a. «Digitales Lernen Grundschule - Ausgangsüberlegungen, Ziele und Strukturen des Entwicklungsprojekts dileg-SL». In Digitale Medien in der Grundschullehrerbildung. Erfahrungen aus dem Projekt dileg-SL, herausgegeben von Thorsten Junge und Horst Niesyto, 17-37. München: kopaed.

Niesyto, Horst. 2019b. «Ergebnisse des Entwicklungsprojekts dileg-SL. Kernpunkte in teilprojektübergreifender Perspektive». In Digitale Medien in der Grundschullehrerbildung. Erfahrungen aus dem Projekt dileg-SL, herausgegeben von Thorsten Junge und Horst Niesyto, 207-232. München: kopaed.

Niesyto, Horst. 2018. «Under Digital Fire. Herausforderungen für die medienpädagogische Professionalisierung». In Futurelab Medienpädagogik. Qualitätsentwicklung - Professionalisierung - Standards, herausgegeben von Thomas Knaus, Dorothee M. Meister, Kristin Narr, 49-69. München: kopaed.

Niesyto, Horst. 2014. "Grundbildung Medien an der Pädagogischen Hochschule Ludwigsburg». In Grundbildung Medien in pädagogischen Studiengängen, herausgegeben von Peter Imort und Horst Niesyto, Schriftenreihe Medienpädagogik interdisziplinär, Band 10, 123-138. München: kopaed. 
Niesyto, Horst. 2010. Handlungsorientierte Medienarbeit. In: Handbuch Mediensozialisation, herausgegeben von Ralf Vollbrecht und Claudia Wegener, 396-403. Wiesbaden: VS Verlag.

Niesyto, Horst, Dorothee M. Meister, und Heinz Moser, Hrsg. 2009. Medien und soziokulturelle Unterschiede. MedienPädagogik: Zeitschrift für Theorie Und Praxis Der Medienbildung 17. https://doi.org/10.21240/mpaed/17.X.

Peschel, Markus, und Thomas Irion, Hrsg. 2016. Neue Medien in der Grundschule 2.O. Grundlagen - Konzepte - Perspektiven. Frankfurt/Main: Grundschulverband.

Rat für Kulturelle Bildung. 2019. Jugend/YouTube/Kulturelle Bildung. Horizont 2019. Eine repräsentative Umfrage unter 12- bis 19-Jährigen zur Nutzung kultureller Bildungsangebote an digitalen Kulturorten. https://www.rat-kulturelle-bildung. de/fileadmin/user_upload/pdf/Studie_YouTube_Webversion_final.pdf.

Reißmann, Wolfgang. 2015. Mediatisierung visuell - Kommunikationstheoretische Überlegungen und eine Studie zum Wandel privater Bildpraxis. Baden-Baden: Nomos.

Rymeš, Robert, und Ulrich Iberer. 2019. «Datenschutzrechtliche Aspekte bei der Durchführung medienbasierter Schulprojekte». In Digitale Medien in der Grundschullehrerbildung. Erfahrungen aus dem Projekt dileg-SL, herausgegeben von Thorsten Junge und Horst Niesyto, 163-176. München: kopaed.

Rymeš, Robert, Jörg-U. Keßler, und Annika Jokiaho. 2019. «Das didaktische Potential von Tablets im Englischunterricht der Grundschule. Ein unterrichtspraktisches Projekt im Rahmen der Lehrer- und Lehrerinnenbildung». In Digitale Medien in der Grundschullehrerbildung. Erfahrungen aus dem Projekt dilegSL, herausgegeben von Thorsten Junge und Horst Niesyto, 131-144. München: kopaed.

Rymeš, Robert, Anja Koppenhöfer, und Jakob Reichel. 2019. «Technische Ausstattungsfragen hinsichtlich der Nutzung mobiler Endgeräte an Schulen und Hochschulen». In Digitale Medien in der Grundschullehrerbildung. Erfahrungen aus dem Projekt dileg-SL, herausgegeben von Thorsten Junge und Horst Niesyto, 177-193. München: kopaed.

Rymeš, Robert, Roland Walter, und Ulrich Iberer, 2019. Datenschutz beim Einsatz digitaler Medien in der Grundschule. Eine Handreichung für Lehrerinnen und Lehrer in Baden-Württemberg mit rechtlichen Grundlagen, pädagogischen Hinweisen und Fallbeispielen, hrsg. vom Projekt dileg-SL. Ludwigsburg: Pädagogische Hochschule. Zugriff 30.11.2019 https://www.ph-ludwigsburg. de/20840+M51093461ac2.html

Schaal, Steffen, und Armin Lude. 2015. «Using mobile devices in environmental education and education for sustainable development - comparing theory and practice in a nation wide survey». Sustainability 7(8), 10153-10170. https://doi. org/10.3390/su70810153.

Schiefner-Rohs, Mandy. 2017. «Medienbildung in Der Schule. Blinde Flecken Und Spannungsfelder in Einer Kultur Der Digitalität». MedienPädagogik: Zeitschrift für Theorie Und Praxis Der Medienbildung 27 (Spannungsfelder \& blinde Flecken), 153-72. https://doi.org/10.21240/mpaed/27/2017.10.15.X. 
Schluchter, Jan-René. 2019. «Methoden inklusiver Medienbildung». In Handbuch Inklusion und Medienbildung, herausgegeben von Ingo Bosse, Jan-René Schluchter und Isabel Zorn, 198-206. Weinheim/Basel: Beltz Juventa.

Schluchter, Jan-René. 2015. Medienbildung als Perspektive für Inklusion - Modelle und Reflexionen für die pädagogische Praxis. München: kopaed.

Schmerr, Martina. 2019. "Zur Rolle der Digitalwirtschaft im Bildungsbereich». medien concret, 1, 58-61.

Schulmeister, Rolf, und Jörn Loviscach. 2017. "Mythen der Digitalisierung mit Blick auf Studium und Lehre». In Digitale Transformation im Diskurs. Kritische Perspektiven auf Entwicklungen und Tendenzen im Zeitalter des Digitalen, herausgegeben von Christian Leineweber und Claudia de Witt. http://nbn-resolving.de/urn:nbn:de:hbz:708-dh5756.

Sektion Medienpädagogik in der Deutschen Gesellschaft für Erziehungswissenschaft. 2017. "Orientierungsrahmen für die Entwicklung von Curricula für medienpädagogische Studiengänge und Studienanteile». MedienPädagogik: Zeitschrift für Theorie Und Praxis Der Medienbildung, (Statements and Frameworks), 1-7. https://doi.org/10.21240/mpaed/00/2017.12.04.X.

Staab, Philipp. 2019. Digitaler Kapitalismus. Markt und Herrschaft in der Ökonomie der Unknappheit. Berlin: Suhrkamp.

Stalder, Felix. 2016. Kultur der Digitalität. Berlin: Suhrkamp.

Thumel, Mareike, und Christina Metzler. 2018. «Teilhabe- und Entwicklungschancen durch aktive Medienarbeit in der Grundschule stärken». medien + erziehung, 6 (2018), 102-112.

Trüby, Daniel. 2017. «Grundbildung Medien an der PH Ludwigsburg - Erste Zwischenbilanz und Evaluation». Ludwigsburger Beiträge zur Medienpädagogik, 17. http://www.medienpaed-ludwigsburg.de/wp-content/uploads/2017/12/Trueby-Grundbildung-Medien-an-der-PH-Ludwigsburg.pdf.

Trüby, Daniel. 2016. Mobiles Lernen an der Schnittstelle von Filmbildung und Sprachförderung. Schriftenreihe Medienpädagogische Praxisforschung, Band 9. München: kopaed.

Tulodziecki, Gerhard, Bardo Herzig, und Silke Grafe. 2019. Medienbildung in Schule und Unterricht. 2. Auflage. Bad Heilbrunn: Julius Klinkhardt.

Verständig, Dan, Alexandra Klein, und Stefan Iske. 2016. "Zero-Level Digital Divide: Neues Netz und neue Ungleichheiten». Si:So. Analysen - Berichte - Kontroversen, 50-55. Universität Siegen. https://nbn-resolving.org/ urn:nbn:de:hbz:467-11973.

Witzke, Margrit. 2004. Identität, Selbstausdruck und Jugendkultur. Eigenproduzierte Videos Jugendlicher im Vergleich mit ihren Selbstaussagen. Ein Beitrag zur Jugend(kultur)forschung. München: kopaed. 